\title{
Soroprevalência de doenças infecciosas em doadores de sangue em um município do Amazonas
}

\author{
Seroprevalence of infectious diseases in blood donors in a municipality in Amazonas
}

Seroprevalencia de enfermedades infecciosas en donantes de sangre en un municipio de Amazonas

Lucelia Barata da Rocha ${ }^{1}$, Josiane Montanho Mariño ${ }^{1}$, Marcelo Henrique da Silva Reis ${ }^{1 *}$, Jéssica Karoline Alves Portugal ${ }^{1}$, Évelyn Janaína da Silva Barão ${ }^{1}$, Duã Louise Aires de Freitas ${ }^{1}$, Ramanda Sena Guimarães ${ }^{1}$, José Carlos Ferreira Pinheiro Junior ${ }^{1}$.

\section{RESUMO}

Objetivo: Analisar a soroprevalência de doenças infecciosas nos doadores de sangue em um município do Amazonas. Métodos: Estudo transversal, descritivo, de análise quantitativa, realizado em um município no interior do Amazonas. Os dados foram obtidos através de consulta aos prontuários dos candidatos à doação no período de janeiro de 2014 a dezembro de 2016, e posteriormente tabulados e analisados com o auxílio do programa Statistical Package for Social Sciences - SPSS. Resultados: Foram analisados 1.590 prontuários de doadores de sangue no período do estudo. Destes, $147(9,25 \%)$ dos candidatos foram considerados inaptos para a doação. Quanto ao perfil epidemiológico dos inaptos, houve uma predominância do sexo masculino $117(79,6 \%)$. A média de idade encontrada foi de 42,52 anos ( $D P=10,04)$. Dentre as doenças pesquisadas, houve a seguinte distribuição: $126(85,7 \%)$ hepatite $B(\mathrm{HBc})$, seguido dos respectivos marcadores: Coinfecção, onde ocorre a infecção por 2 ou mais patógenos, foram encontrados 9 casos $(6,1 \%)$, Sífilis (VDRL) 6 (4,1\%), Anti-HCV $3(2,0 \%)$, doença de Chagas $2(1,4 \%)$ e HBsAG 1 (0,06\%). Conclusão: A pesquisa demonstrou que a triagem sorológica é essencial entre doadores de sangue, visto que ela pode identificar a presença de infecções e posteriormente evitar que ocorra a contaminação de outras pessoas.

Palavras-chave: Doadores de sangue, Doenças infecciosas, Bancos de sangue.

\begin{abstract}
Objective: To analyze the seroprevalence of infectious diseases in blood donors in a municipality in Amazonas. Methods: Cross-sectional, descriptive, quantitative analysis study carried out in a municipality in the interior of Amazonas. The data were obtained by consulting the medical records of the candidates for the donation from January 2014 to December 2016, and subsequently tabulated and analyzed with the aid of the Statistical Package for Social Sciences - SPSS program. Results: 1,590 blood donor records were analyzed during the study period. Of these, $147(9.25 \%)$ of the candidates were considered unfit for donation. Regarding the epidemiological profile of the disabled, there was a predominance of males 117 (79.6\%). The average age found was 42.52 years $(S D=10.04)$. Among the diseases surveyed, there was the following distribution: 126 $(85.7 \%)$ hepatitis $B(\mathrm{HBc})$, followed by the respective markers: Coinfection, where infection by 2 or more pathogens occurs, 9 cases (6.1\%) were found, Syphilis (VDRL) 6 (4.1\%), Anti-HCV 3 (2.0\%), Chagas disease $2(1.4 \%)$ and HBsAG $1(0.06 \%)$. Conclusion: Research has shown that serological screening is essential among blood donors, as it can identify the presence of infections and subsequently prevent other people from becoming infected.
\end{abstract}

Keywords: Blood donors, Infectious diseases, Blood banks.

1 Universidade Federal do Amazonas (UFAM), Coari - AM. * E-mail: reis.henrique.marcelo@gmail.com SUBMETIDO EM: 5/2020 ACEITO EM: 6/2020 PUBLICADO EM: $\mathbf{8 / 2 0 2 0}$ 


\section{RESUMEN}

Objetivo: analizar la seroprevalencia de enfermedades infecciosas en donantes de sangre en un municipio de Amazonas. Métodos: Estudio de análisis transversal, descriptivo, cuantitativo, realizado en un municipio del interior del Amazonas. Los datos se obtuvieron consultando los registros médicos de los candidatos a la donación de enero de 2014 a diciembre de 2016, y posteriormente se tabularon y analizaron con la ayuda del paquete estadístico para el programa SPSS. Resultados: se analizaron 1,590 registros de donantes de sangre durante el período de estudio. De estos, 147 (9,25\%) de los candidatos fueron considerados no aptos para la donación. En cuanto al perfil epidemiológico de los discapacitados, predominó el sexo masculino 117 $(79,6 \%)$. La edad promedio encontrada fue de 42.52 años $(D E=10.04)$. Entre las enfermedades encuestadas, hubo la siguiente distribución: $126(85.7 \%)$ hepatitis B (HBc), seguida de los marcadores respectivos: Coinfección, donde ocurre la infección por 2 o más patógenos, se encontraron 9 casos $(6.1 \%)$, Sífilis (VDRL) $6(4,1 \%)$, anti-VHC $3(2,0 \%)$, enfermedad de Chagas $2(1,4 \%)$ y HBsAG 1 (0,06\%). Conclusión: La investigación ha demostrado que la detección serológica es esencial entre los donantes de sangre, ya que puede identificar la presencia de infecciones y, posteriormente, evitar que otras personas se infecten.

Palabras clave: Donantes de sangre, Enfermedades infecciosas, Bancos de sangre.

\section{INTRODUÇÃO}

O processo de hemoterapia é uma prática rotineira de transfusão de sangue amplamente utilizada em todo o mundo, que consiste no tratamento terapêutico realizado através da transfusão sanguínea, de seus componentes ou derivados. Trata-se de uma atividade assistencial de alto risco epidemiológico, uma vez que o sangue, na condição de tecido vivo, é capaz de transmitir diversas doenças (BRASIL, 2013).

No mundo, a transfusão de sangue destaca-se em dois períodos, dentre eles, enfatizamos: período empírico, que vai até o ano de 1900 e período científico, que vai de 1900 até os dias atuais. Em âmbito nacional, a prática transfusional ainda está em fases de evolução, assim como acontece em outros países do globo. No século XX, grandes metrópoles como Rio de Janeiro e São Paulo lideraram a evolução da hemoterapia no país, além destas capitais, outras cidades como Bahia, Pernambuco e Porto Alegre, tiveram grandes papéis de destaque neste processo de evolução (JUNQUEIRA PC, et al., 2005).

A fim de garantir uma prática segura no processo de transfusão sanguínea, alguns fatores precisam ser levados em consideração, visto que em conjunto, eles serão capazes de determinar a qualidade dos hemocomponentes que serão transfundidos. Dentre esses importantes fatores, podemos destacar: a triagem sorológica, triagem clínica, realização dos testes imunohematológicos, além disto, a seleção da população de doadores também é primordial (MARCA F e WEIDLICH L, 2016).

A transfusão de sangue pode ser uma intervenção que salva vidas, no entanto, como todos os tratamentos, engloba diversos riscos à saúde do indivíduo, em seu processo de evolução muitas pessoas que necessitavam desse meio para tratar determinadas patologias foram contaminadas ao longo dos anos, neste contexto, adquiriram doenças oriundas do sangue transfundido. A transmissão de doenças através do sangue é manifestada mesmo antes da existência dos primeiros bancos de sangue, entretanto, na década de 80 com o surgimento da epidemia do vírus da imunodeficiência humana (HIV), houve uma importante revolução nos serviços responsáveis pelo processo de hemoterapia em todo o planeta, visto que foi comprovado que a doença tinha transmissão pelo sangue (SANTOS EA, et al., 2008).

Seguindo as recomendações do Ministério da Saúde, cujo intuito é a garantia que o sangue utilizado nas transfusões seja capaz de oferecer o risco mínimo ao doador, o candidato a doação necessita passar pela triagem clínica e sorológica, neste contexto, apenas os candidatos aptos na triagem clínica serão encaminhados à triagem sorológica. As medidas pré-transfusionais, como a captação e seleção de doadores, somadas aos testes de triagem sorológica, diminuem sensivelmente a possibilidade de transmissão de doenças por meio de transfusão (ROHR Jl, et al., 2012). 
Previamente ao processo de transfusão, o sangue total e todos os seus componentes necessitam passar pela triagem, que é responsável por detectar as seguintes doenças: hepatite B (anti HBV e HBsAg), hepatite C (anti-HCV), HIV-1 e HIV-2 (anti-HIV e HIV Ag/Ac), doença de Chagas, Sífilis (VDRL), vírus linfotrópico humano tipo I e II (anti-HTLV e HTLV-II). Após essa verificação, caso haja obtenção de positividade, o possível doador deverá ser excluído de forma temporária ou definitiva, dependendo da situação, esse indivíduo precisará ser encaminhando a um serviço de referência (BRASIL, 2013; BRASIL, 2014).

No estado do Amazonas, o processo de hemoterapia enfrenta desafios particulares devido às diversidades epidemiológicas e geográficas regionais de alto impacto na logística do abastecimento e da manutenção dos estoques de hemoderivados. Todas as amostras de sangue dos doadores do estado são submetidas a exames laboratoriais de triagem na Fundação de Hematologia e Hemoterapia do estado do Amazonas, situada em Manaus, onde funciona a central sorológica (FHEMOAM, 2010).

De acordo com informações do Ministério da saúde, em 2015, no Brasil e na região norte, a taxa de descarte por inaptidão sorológica nos bancos de sangue foi de $17,62 \%$ e $20,86 \%$, respectivamente. Esse índice é mais alto do que nos países desenvolvidos, principalmente devido à alta porcentagem de pessoas que doam sangue pela primeira vez e que apresentam uma prevalência de infecção próxima à da população em geral (BRASIL, 2017).

Uma das principais causas da inaptidão sorológica nos bancos de sangue é devido a positividade para o vírus da Hepatite B. Esse problema torna-se ainda mais alarmante quando relacionados à população do Estado do Amazonas, uma vez que a região Amazônica é uma das regiões do mundo considerada endêmica para Hepatites virais, além de outras doenças infecciosas (FONSECA JCF, 2010).

A carência de dados epidemiológicos no Brasil, e mais especificamente na região Norte, serve como subsídio para a realização deste estudo, buscando maior conhecimento sobre características desta população através do levantamento de dados sobre as doenças infecciosas dos doadores de sangue de um município do Amazonas. Diante disto, este estudo tem como objetivo analisar a soroprevalência de doenças infecciosas nesses doadores, bem como verificar o perfil epidemiológico dos doadores de sangue com resultado sorológico positivo para doenças infecciosas em relação à idade e sexo.

\section{MÉTODOS}

Trata-se de um estudo transversal, de análise quantitativa, caráter descritivo, conduzido através da consulta aos prontuários de candidatos à doação de sangue de um município do Amazonas, realizada por acadêmicos de enfermagem da Universidade Federal do Amazonas, entre janeiro de 2014 a dezembro de 2016.

Foram incluídos no estudo, prontuários de pacientes de ambos os sexos, com idades a partir de 18 anos, que apresentavam resultados de exames positivos para testes sorológicos. Se enquadraram nos critérios de exclusão os prontuários com registro de resultados de exames de pacientes sem alterações, que estavam inelegíveis e de difícil compreensão.

Para obtenção das informações de seguimento, foi realizado um levantamento dos dados utilizando os prontuários de doadores de sangue que apresentaram resultados positivos para testes sorológicos. Foram retiradas as seguintes informações: marcador sorológico, idade e sexo.

Os dados foram tabulados e analisados com o auxílio do programa Statistical Package for Social Sciences (SPSS) versão 20.0 for Windows. Os resultados foram apresentados por meio de tabelas, sendo que na análise das variáveis quantitativas, quando os dados apresentavam distribuição normal, foi calculada a média e o desvio-padrão (DP), em relação às variáveis categóricas, foram analisadas pelo número e percentual, através do cálculo das frequências absolutas simples e relativas, e em alguns casos o Intervalo de Confiança (IC) ao nível de 95\%.

O estudo foi submetido e aprovado pelo Comitê de Ética em Pesquisa da Universidade Federal do Amazonas, com protocolo de no 66459417.0.0000.5020. 


\section{RESULTADOS}

No período de 2014 a 2016 foram analisados 1.590 prontuários de candidatos a doações voluntárias de sangue realizadas em um município do Amazonas. Destes, $1.443(90,75 \%)$ foram considerados aptos nas amostras para os testes sorológicos obrigatórios, e 147 (9,25\%) dos candidatos foram considerados inaptos para a doação (Tabela 1).

A principal causa de inaptidão das amostras foi o resultado positivo para alguns marcadores sorológicos, onde a mais elevada prevalência foi a presença do vírus da hepatite B (HBV) com 126 (85,7\%) dos resultados positivos, seguido dos respectivos marcadores: Coinfecção, onde ocorre a infecção por 2 ou mais patógenos, na amostra foram encontrados 9 casos (6,1\%), Sífilis ( VDRL) $6(4,1 \%)$, Anti-HCV foi de $3(2,0 \%)$, doença de Chagas $2(1,4 \%)$ e HBsAG de $1(0,06 \%)$. Para os marcadores Anti-HIV e HIVAg/Ac não houve resultados positivos na amostra. Todos os dados citados estão bem representados na Tabela 1.

Tabela 1 - Prevalência de doações inaptas por sorologia em um município do Amazonas, no período de 2014 a 2016.

\begin{tabular}{ccc}
\hline Doença & $\begin{array}{c}\text { Prevalência em relação ao total } \\
\text { de doadores }\end{array}$ & $\begin{array}{c}\text { Prevalência em relação ao número de } \\
\text { doadores inaptos }\end{array}$ \\
\hline HBc & $7,92 \%$ & $126(85,7 \%)$ \\
Coinfecção & $0,57 \%$ & $9(6,1 \%)$ \\
Sífilis (VDRL) & $0,38 \%$ & $6(4,1 \%)$ \\
Anti-HCV & $0,19 \%$ & $3(2,0 \%)$ \\
Doença de Chagas & $0,13 \%$ & $2(1,4 \%)$ \\
HBsAg & $0,06 \%$ & $1(0,7 \%)$ \\
\hline Total & 1590 & 147 \\
\hline
\end{tabular}

Fonte: Rocha LB, et al., 2020.

Dos inaptos, 9 casos (100\%) foram encontrados com duas ou mais infecções, destes, destacamos: HBc+Sífilis $4(44,4 \%), \mathrm{HBc}+\mathrm{HBsAg} 2(22,2 \%), \mathrm{HBc}+\mathrm{HCV} 2(22,2 \%)$ e HBc+Sífilis+Chagas 1 (11,2\%). Em relação aos doadores inaptos, os indivíduos do sexo masculino foram mais frequentes, representando 117 $(79,6 \%)$ dos doadores, enquanto o sexo feminino representou $30(20,4 \%)$ dos doadores (Tabela 2$)$.

Tabela 2 - Soroprevalência por sexo dos doadores de sangue em um município do Amazonas, no período de 2014 a 2016.

\begin{tabular}{|c|c|c|c|c|c|c|}
\hline \multirow{2}{*}{ Marcadores } & & \multicolumn{4}{|c|}{ Sexo } & \multirow{2}{*}{ Total $(n=147)$} \\
\hline & & Masculino $(n=117)$ & $\%$ & Feminino $(n=30)$ & $\%$ & \\
\hline \multirow{2}{*}{ Anti-HBc } & $\mathrm{R}$ & 101 & 68,7 & 25 & 17,0 & 126 \\
\hline & NR & 9 & 6,1 & 3 & 2,0 & 12 \\
\hline \multirow{2}{*}{ HBsAg } & $\mathrm{R}$ & 1 & 0,8 & 0 & 0,0 & 1 \\
\hline & NR & 109 & 74,1 & 28 & 19,0 & 137 \\
\hline \multirow{2}{*}{$\begin{array}{l}\text { Sífilis } \\
\text { (VDRL) }\end{array}$} & $\mathrm{R}$ & 5 & 3,4 & 1 & 0,7 & 6 \\
\hline & NR & 105 & 71,4 & 27 & 18,4 & 132 \\
\hline \multirow{2}{*}{ Anti_HCV } & $\mathrm{R}$ & 2 & 1,4 & 1 & 0,8 & 3 \\
\hline & NR & 108 & 73,3 & 27 & 18,4 & 135 \\
\hline \multirow{3}{*}{$\begin{array}{l}\text { Doença de } \\
\text { Chagas } \\
\text { Coinfecção }\end{array}$} & $\mathrm{R}$ & 1 & 0,7 & 1 & 0,7 & 2 \\
\hline & NR & 109 & 74,1 & 27 & 18,4 & 136 \\
\hline & $\mathrm{R}$ & 7 & 4,8 & 2 & 1,4 & 9 \\
\hline
\end{tabular}

Legenda: $\mathbf{R = r e a g e n t e , ~} \mathbf{N R = n a ̃ o ~ r e a g e n t e ; ~ F o n t e : ~ R o c h a ~ L B , ~ e t ~ a l . , ~} 2020$.

Quanto à distribuição da faixa etária dos doadores de sangue nesse período, observou-se que a média de idade dos indivíduos com amostra positiva foi de 42,52 anos ( $D P=10,04)$, com prevalência na faixa etária de 31 a 50 anos (Tabela 3). 
Tabela 3 - Distribuição da idade em relação à inaptidão pelas doenças transmissíveis em doadores de sangue em um município do Amazonas, no período de 2014 a 2016.

\begin{tabular}{|c|c|c|c|c|c|c|c|}
\hline \multirow{2}{*}{ Marcadores } & & \multicolumn{5}{|c|}{ Idade } & \multirow{2}{*}{ Total } \\
\hline & & 20 a 30 & 31 a 40 & 41 a 50 & 51 a 60 & 61 a 78 & \\
\hline \multirow{2}{*}{ Anti-HBc } & $\mathrm{R}$ & 11 & 45 & 41 & 26 & 3 & 126 \\
\hline & NR & 5 & 2 & 2 & 2 & 1 & 12 \\
\hline \multirow{2}{*}{$\mathrm{HBsAg}$} & $\mathrm{R}$ & 1 & 0 & 0 & 0 & 0 & 1 \\
\hline & NR & 15 & 47 & 43 & 28 & 4 & 137 \\
\hline \multirow{2}{*}{ Anti_HCV } & $\mathrm{R}$ & 3 & 0 & 0 & 0 & 0 & 3 \\
\hline & NR & 13 & 47 & 43 & 28 & 4 & 135 \\
\hline \multirow{2}{*}{$\begin{array}{l}\text { Doença de } \\
\text { Chagas }\end{array}$} & $\mathrm{R}$ & 1 & 0 & 0 & 1 & 0 & 2 \\
\hline & NR & 15 & 47 & 43 & 27 & 4 & 136 \\
\hline \multirow{2}{*}{$\begin{array}{l}\text { Sífilis } \\
\text { (VDRL) }\end{array}$} & $\mathrm{R}$ & 0 & 2 & 2 & 1 & 1 & 6 \\
\hline & NR & 16 & 45 & 41 & 27 & 3 & 132 \\
\hline Coinfecção & $\mathrm{R}$ & 1 & 4 & 3 & 1 & 0 & 9 \\
\hline
\end{tabular}

Fonte: Rocha LB, et al., 2020.

\section{DISCUSSÃO}

A verificação da segurança das transfusões de sangue tem início quando ocorre o recrutamento dos eventuais doadores através da triagem clínica. Em relação ao índice de inaptidão sorológica para os marcadores de doenças transmissíveis pelo sangue encontrado no presente estudo, obteve-se o resultado $(9,25 \%)$, que é inferior quando comparado ao índice encontrado no Brasil e na região norte, onde a taxa de descarte sorológico foi de 17,62\% e 20,86\%, respectivamente (BRASIL, 2017).

A prevalência de uma determinada infecção não possui representação pela taxa de descarte sorológico, apesar disto, a taxa reflete um conjunto de variáveis que têm grande relevância para avaliar a qualidade do sangue a ser transfundido (FERREIRA DM, et al., 2012).

Com relaçao a soroprevalência de doenças encontradas na presente pesuisa, o maior número de casos da infecção foi pelo vírus da hepatite $B$ em indivíduos do sexo masculino, com idades que variaram de 31 a 50 anos. A Hepatite B é mais prevalente em doadores de sangue mais velhos e do sexo masculino, e o tempo de exposição é considerado um dos fatores que interferem no incremento da infecção pelo vírus da hepatite B (COSTA LG, et al., 2008; MARTINS APB, et al., 2015).

A predominância do sexo masculino na pesquisa pode estar relacionada a aspectos comportamentais de risco. Além disto, os homens procuram mais os serviços de doação de sangue quando comparado às mulheres (BRASIL, 2013; IVA J, 2010). Situação semelhante a esta foi encontrada num estudo realizado no Hemocentro Regional de Cruz Alta - Rio Grande do Sul, onde o descarte das bolsas foi maior entre o sexo masculino, constituindo de $61,1 \%$ dos casos e também em estudo oriundo da Índia (MARTINS APB, et al., 2015; BASAK S, et al., 2017).

Os dados do presente estudo corroboram com a pesquisa realizada no centro de triagem de doadores voluntários do município de Paulo Afonso - Bahia, no período de 2009 a 2011, onde observou-se uma predominância $(54,7 \%)$ de indivíduos do sexo masculino com idades ente 42 a 49 anos e infectados pelo vírus da hepatite $B$ (HBV) (SALGADO APS, et al., 2016).

A projeção global da Organização Mundial da Saúde (OMS) de 2015 para os casos de infecção por Hepatite B é de 3,5\% da população do planeta, aproximadamente 257 milhões de pessoas (WHO, 2017). Neste panorama, é importante destacar que para a hepatite $B$, são utilizados dois marcadores sorológicos: 0 antígeno HBsAg e o anticorpo anti-HBc. O HBsAg é um soromarcador para hepatite B que indica infecção aguda ou crônica. $\mathrm{O}$ anti-HBc, por sua vez, é um marcador detectável durante todos os estágios da infecção pelo vírus da hepatite $\mathrm{B}$, exceto na fase inicial da exposição viral. Este marcador persiste na infecção, mesmo após o HBsAg desaparecer, bem como no estágio portador crônico (BRASIL, 2016). 
Os bancos de sangue da região Norte registram um percentual de inaptidão sorológica para anti-HBc de $2,26 \%$, enquanto para o HBsAg de 2,30 \%. Ao se comparar os valores percentuais de inaptidão sorológica da região Norte com outras regiões do Brasil, observa-se uma elevada prevalência da Hepatite B: Região nordeste $(1,59 \%)$, Centro Oeste $(1,80 \%)$, Sudeste $(1,26 \%)$ e Sul $(1,36 \%)$ dos casos (FERREIRA DM, et al., 2012).

Segundo informações do Ministério da Saúde (MS), este dado é mais alarmante para o Estado do Amazonas, onde o percentual de inaptidão sorológica para os marcadores Anti-HBc e HBsAg testados nos bancos de sangue foi de $2,42 \%$ e 17,23\% simultaneamente, indicando uma elevada prevalência da hepatite B em doadores de sangue, com intensa circulação do vírus na região amazônica (BRASIL, 2017).

A amostra apontou $(6,11 \%)$ de coinfecção entre os casos inaptos, resultado similar ao encontrado em outros estudos (PEDROSO D, et al., 2016; CAO WW, et al., 2018). Este achado apresenta grande impacto, visto que com a presença de doenças simultâneas, ambas as patologias atuando em conjunto tendem a acometer consideravelmente o quadro clínico do indivíduo. Neste contexto, é primordial que haja a comunicação entre a rede de referência e contrarreferência para acompanhamento dos casos.

Além da infecção pelo vírus da Hepatite $B$, outros marcadores sorológicos foram responsáveis pela exclusão da bolsa de sangue neste trabalho como: Sífilis, Hepatite $C$ e Doença de Chagas. Durante o período estudado, a frequência de doadores com sorologia reativa para sífilis, foi de $(4,1 \%)$, dado superior ao encontrado na África $(3,1 \%$ ) (OKOROIWU HU, et al., 2018). A sífilis é uma doença infecciosa crônica causada pelo Treponema pallidum e em alguns países, como o Brasil, é considerada como um dos principais problemas de saúde pública, similar a outras doenças infecciosas emergentes no país (AVELLEIRA JCR, BOTTINO G, 2006; SOUZA EM, 2005).

De acordo com a Organização Mundial de Saúde, a estimativa é de aproximadamente 12 milhões de novos casos de pessoas infectadas por ano com alguma doença relacionada ao sexo, dentre as quais a sífilis apresenta grande representatividade (BRASIL, 2006). Tais dados se assemelham aos obtidos pela Agencia Nacional de Vigilância Sanitária (ANVISA) que encontrou prevalência mais elevadas nos bancos de sangue para os marcadores de Sífilis $(0,76 \%)$ e Hepatite B $(1,57 \%)$ quando comparado a outros marcadores sorológicos (BRASIL, 2015).

A prevalência de sífilis $(4,1 \%)$ e da hepatite $B(85,7 \%)$ juntas demonstraram um elevado índice de inaptidão sorológica. Tais dados, corroboram com o estudo realizado no Hemocentro Regional de Montes Claros-MG, no período de 2009 a 2013, onde os testes sorológicos reativos confirmaram elevada inaptidão sorológica pelos marcadores Anti-HBc $(44,4 \%)$ e Sífilis (23,9\%) (MAGALHÃES TA, et al., 2016).

No contexto geral, dentre todos os resultados, o marcador de hepatite B apresentou prevalência de 7,92\%, dado superior ao encontrado em pesquisas no continente Africano, realizada no Gabão, que apresentou 7,28\% e Nigéria com 4,1\% (MBA JME, et al., 2018; OKOROIWU, et al., 2018). Porém, inferior a outros estudos também realizados na África, com os respectivos percentuais: 14,5\%, 19,08\% e 13,3\%, dados oriundos das populações de Burkina Faso, Togo e Gana (TAO I, et al., 2014; KOLOU M, et al., 2017; RUFAI T, et al., 2014).

Essa predominância evidenciada da presença do vírus da hepatite $\mathrm{B}$ e da sífilis, como as principais patologias associadas a triagem sorológica podem estar associadas ao fato de que, atualmente, a população exerce um efeito cumulativo de riscos comportamentais. Estes envolvem, principalmente, o sexo sem proteção, já que estas duas doenças são transmitidas principalmente através da relação sexual, o uso de drogas ilícitas injetáveis e outras exposições a sangue e hemoderivados (CRISTINA L, et al., 2013; MBA JME, et al., 2018).

No ano de 2015, a OMS apontou que aproximadamente 1\% da população mundial viva com o vírus da hepatite $\mathrm{C}$, um dado preocupante, pois se trata de uma doença que se não for manejada da maneira correta tende a trazer grandes complicações ao portador (WHO, 2017).

Em relação à infecção por HCV, no presente estudo, a prevalência de doações de sangue inaptas para o HCV foi responsável pelo descarte de 2,0\%. Taxa superior quando comparada a estudos realizados nos 
estados e municípios brasileiros sobre a taxa de prevalência de anti- HCV, em doadores de sangue, onde observou-se as seguintes variações: $0,4 \%$ no Hemocentro regional de Uberaba/MG, 0,44\% no Hemonúcleo de Tucuruí/PA - HENT, além de ser maior que a taxa encontrada na China $(1,43 \%)$ (JOSAHKIAN JA, et al., 2010; BARROS EC, JUNIOR LCB, 2012; CAO WW, et al., 2018).

Ainda relacionado ao marcador de HCV, a taxa do estudo foi inferior as encontradas pela Fundação de Hematologia e Hemoterapia do estado do Amazonas - FHEMOAM/AM, que apresentou 9,0\% e em estudos realizados no continente Africano, que apontou taxas de 10,98\% e 3,6\%, respectivamente (OLIVEIRA AA, et al., 2009; MBA JME, et al., 2018; OKOROIWU HU, et al., 2018).

Como pode ser observado, há uma variação das taxas de incidência encontradas em outros estudos, o que pode ser devido às diferenças da metodologia empregada, variação das condições socioeconômicas e epidemiológicas da população de cada região, ao índice de fidelização dos doadores, ao uso de técnicas sorológicas diversas e ao tamanho das amostras de cada estudo.

Na região Amazônica, o desmatamento desordenado da floresta, as migrações populacionais humanas de áreas endêmicas e o grande número de reservatórios e vetores silvestres naturalmente infectados por Trypanosoma cruzi favorece a endemicidade da doença de Chagas nessa região, com uma prevalência sorológica geral de 1,2\% e de 0,9\% para o Estado do Amazonas (MARIA RL e REIKDAL OS, 2003).

Em relação ao marcador sorológico para Doença de Chagas, apesar de a região amazônica ser considerada endêmica, foi encontrado uma baixa prevalência $(1,4 \%)$ de doações inaptas. Uma hipótese plausível para a soroprevalência da doença de Chagas no presente estudo seria a existência de um número maior de indivíduos que ainda não foram detectados pelo Sistema Único de Saúde, em virtude da ausência de sintomatologia nos portadores.

O percentual de Doença de Chagas $(1,4 \%)$ é superior a estudo da cidade de Porto Alegre, dos autores Fitarelli DB e Horn JF (2008) e inferior a estudo de um município do Rio Grande do Sul, que apresentou 2,7\% de sororreatividade (PEDROSO D, et al., 2016). Segundo dados da ANVISA, esses percentuais distanciamse da taxa média nacional do marcador entre candidatos à doação (0,26\%) (ANVISA, 2013).

Em outro estudo realizado para verificar a taxa de prevalência de Doença de Chagas em doadores de sangue observou-se que a taxa de descarte oscilou entre $5,1 \%$ a $12,1 \%$ no Núcleo de Hemoterapia na cidade Três Lagoas/MS (BORELLI SD, et al., 2013). Já em um outro estudo realizado no Serviço de Hemoterapia do Hospital Universitário de Santa Maria (HUSM) na Região Sul, entre janeiro de 2004 a dezembro de 2007, observou uma prevalência de $0,98 \%$, da doença (PIVETTA J, et al., 2014).

\section{CONCLUSÃO}

O presente estudo apontou que 9,25\% $(n=147)$ dos doadores de sangue envolvidos na análise foram considerados inaptos por apresentarem algum marcador sorológico positivo. Em relação ao sexo, os homens foram os mais acometidos, representando $79,6 \%(n=117)$ dos casos, comparado ao sexo feminino. A faixa etária de maior acometimento foi em indivíduos de 31 a 50 anos. Algumas limitações no presente estudo devem ser apontadas. Não foi possível analisar de forma mais completa as variáveis sociodemográficas dos doadores, considerando-se o resguardo da confidencialidade dos dados, além disto, a pesquisa demonstrou que a triagem sorológica é essencial entre doadores de sangue, visto que ela pode identificar a presença de infecções e posteriormente evitar que ocorra a contaminação de outras pessoas.

\section{REFERÊNCIAS}

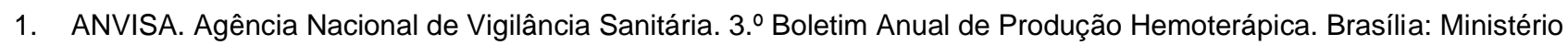
da Saúde. 2013.

2. BARROS EC, JUNIOR LCB. Perfil epidemiológico de doadores de sangue inaptos por sorologia para hepatite $\mathrm{c} 1$. Intituto Ciência Biol. 2012. 
3. BASAK S, et al. Comparison of seropositivity of human immunodeficiency vírus, hepatites $B$ vírus, hepatites $\mathrm{C}$ vírus, and syphilis among hospital córnea retrieval programme-donors versus voluntary cornea denors at a large eye bank in eastern India. Indian Journal of ophthalmology. 2017.

4. BORELLI SD, et al. Blood discard rate and the prevalence of infectious and contagious diseases in blood donors from provincial towns of the state of Paraná, Brazil. Rev Bras Hematol Hemoter, 2013.

5. BRASIL. Diagnótico de Hepatites Virais. Ministério da Saúde Secr Vigilância em Saúde Dep DST, Aids e Hepatites Virais Univ Fed St Catarina. 2016.

6. BRASIL. Ministério da Saúde Agência Nacional de Vigalância Sanitária. Relatório dos dados da produção hemoterápica Bras - Hemoprod 2013. 2015.

7. BRASIL. Ministério da Saude. 4o Bioletim Produção Hemoterápica - Hemoprod 2014 e 2015. 2017.

8. BRASIL. Ministerio da Saúde. Cad informação sangue e hemoderivados dados 2015. 2017.

9. BRASIL. Ministério da Saúde. Caderno de Informação: sangue e hemoderivados: rede física, produção, gastos públicos com hemoterapia, consumo de hemoderivados [Internet]. Brasília: Ministério da Saúde; 2014.

10. BRASIL. Ministério da Saúde. Portaria n. 2.712, de 12 de novembro de 2013. Redefine o regulamento técnico de procedimentos hemoterápicos. Diário Oficial da União. Brasília: Ministério da Saúde; 2013.

11. BRASIL. Ministério da sáude. Portaria no 158, 04 fevereiro 2016 DOU 05/02/2016. 2016.

12. BRASIL. Ministério da Saúde. Relatório dos dados da produção hemoterápica Bras - Hemoprod 2013. 2015;2(Agência Nacional de Vigilância Sanitária):120.

13. BRASIL. Ministério da Saúde. Secretaria de Vigilância em Saúde. Programa Nacional de DST e Aids. Man Control das Doenças Sex Transm. 2006.

14. CAO WW, et al. Prevalence of hepatites B vírus, hepatites $C$ vírus, human immunodeficiency vírus and treponema pallidum infections in hospitalized patients before transfusion in xiangya hospital central South university, China from 2011 to 2016 . BMC Infectious diseases. 2018.

15. COSTA, LG, et al. Detecção de infecção pelo vírus da hepatite B nos municípios brasileiros segundo cobertura dos serviços de hemoterapia, no período de 2001 a 2008*. 2008.

16. CRISTINA L, et al. Soroprevalência do descarte de bolsa de sangue em um Núcleo de Hemoterapia de Três LagoasMS. CESUMAR. 2013.

17. FERREIRA DM, et al. Análise dos aspectos epidemiológicos, hematológicos e sorológicos presentes em doadores de sangue do Hemocentro Regional de Cruz Alta. Bras Rev Bras Soc. 2012.

18. FHEMOAM. Fundação de Hematologia e Hemoterapia do estado do Amazonas Gerencia da Sorologia. 2010.

19. FONSECA JCF. Histórico das hepatites virais. Rev Soc Bras Med Trop. 2010.

20. IVA J. Perfil dos candidatos inaptos para doação de sangue no serviço de hemoterapia do hospital Santo Ângelo, RS, Brasil. Rev Patol Trop. 2010.

21. JCR, BOTTINO G. Sífilis: Diagnóstico, tratamento e controle. An Bras Dermatol. 2006.

22. JOSAHKIAN JA, et al. Prevalência de inapdão sorologica pelo vírus HVC em doadores de sangue no hemocentro regionak de Umberaba MG Fundação Hemominas. Rev Patol Trop. 2010.

23. JUNQUEIRA PC, et al. História da Hemoterapia no Brasil. Rev Bras Hematol Hemoter. 2005.

24. KOLOU M, et al. High Prevalence of Hepatitis B Virus Infection in the Age Range of 20-39 Years Old Individuals in Lome. The open virology journal. 2017.

25. MAGALHÃES TA, et al. Prevalência de inaptidão sorológica dos doadores de sangue no hemocentro regional de Montes Claros, Minas Gerais. Rev Fund Care Online. 2016.

26. MARCA F, WEIDLICH L. Soroprevalência em doadores de sangue do Vale do Taquari, RS. RBAC. 2016.

27. MARIA RL, REIKDAL OS. A família e a enfermeira no contexto domiciliar: dois lados de uma realidade. BDENF. 2003.

28. MARTINS APB, et al. Soroprevalência de doenças infecciosas em doadores de sangue do hemocentro regional de Cruz Alta-Rio Grande do Sul. Clin Biomed Res. 2015.

29. MBA JME, et al. Prevalent hepatites B surface antigen among first-time blood donor in gabon. PLoS ONE 13 (4). 2018.

30. OKOROIWU HU, et al. Seroprevalence of transfusion-transmissible infections (HBV, HCV, syphilis and HIV) among prospective blood donors in a tertiary health care facility in Calabar, Nigeria; an eleven years evaluation. BMC Public Health, 2018.

31. OLIVEIRA AA, et al. Hepatitis C Virus in Blood Donors, Brazil. Emerg Infect Diaseases. 2009.

32. PIVETTA J, et al. Soropositivos para Doença de Chagas na Região Sul. Saúde. 2014.

33. ROHR JI, et al. Perfil dos candidatos inaptos para doação de sangue no serviço de hemoterapia do Hospital Santo Ângelo, RS, Brasil. Rev Pat Trop. 2012.

34. RUFAI T, et al. The prevalence of hepatitis B virus E antigen among Ghanaian blood donors. The Pan African medical journal. 2014.

35. SALGADO APS, et al. Ocorrência da sorologia positiva para hepatite B nos doadores de sangue do Municipio de São Paulo Afonso, Bahia, Brasil, No periodo de 2009 a 2011. Rev Saúde Física Ment. 2014.

36. SANTOS EA, et al. Avaliação epidemiológica das rejeições dos doadores de sangue no HEMOLACEN/SE no Período de 2004 a 2006. Rev. Bras. Analises Clinicas [periódico na Internet]. Rio de Janeiro, 2008.

37. SOUZA EM. Há 100 anos, a descoberta do Treponema pallidum. An Bras Dermatol. 2005.

38. TAO I, et al. Seroepidemiology of hepatitis B and C viruses in the general population of burkina faso. Hepatitis research and treatment. 2014.

39. World Health Organization (WHO). Global hepatitis report 2017. Geneva: World health Organization. p. 2017. 